JSRM Code: 013010400005

\title{
Comparative study of the methods of extracting mesenchymal stem cells from cryopreserved Wharton's Jelly
}

\author{
Boey PYK ${ }^{1 \#}$, Lim DSL ${ }^{1 \#}$, Tang $\mathrm{KF}^{1}, \mathrm{Li} \mathrm{MM}^{1}$, Ekaputra AK ${ }^{1}$, Chowdhury $\mathrm{PK}^{1}$, Mukherjee RAG ${ }^{1}$, Teo $\mathrm{J}^{1}$, Faundo AC ${ }^{1}$, Chiew $^{1} \mathrm{~F}^{1}$
}

\begin{abstract}
The Wharton's Jelly (WJ) is an established source of mesenchymal stem cells (MSC). We compared 3 methods of extracting WJ-MSC from cryopreserved tissue and determined that enzymatic digestion of the WJ yielded the most viable MSC, compared to the explant and mechanical digestion methods. The enzymatically-released WJ-MSC conformed to the International Society for Cellular Therapy (ISCT) criteria: displayed plastic-adherence, co-expressed CD73, CD90, CD105 and were negative for hematopoietic lineage cell markers.
\end{abstract}

Key Words: Mesenchymal stem cell, Wharton's Jelly

Mesenchymal Stem Cells (MSC) are multipotent stromal cells first reported to be found in adult bone marrow $(\mathrm{BM})^{[1]}$, with enormous therapeutic potential because of their ability to differentiate into cells of the adipogenic, osteogenic and chondrogenic lineages ${ }^{[2]}$ Cultured MSC are well-characterized to be plastic-adherent and spindle-shaped, express a panel of cell surface markers including CD73, CD90, CD105, HLA-ABC, and are absent for CD31, CD34, CD45, CD117 and HLA-DR ${ }^{[3]}$. MSC are also found in the umbilical cord (UC) and preferred over BM-MSC because of their fetal origin, greater capacity for proliferation and propensity for trilineage differentiation $^{[4]}$.

As UC-MSC usually do not experience immediate autologous utility, it may be prudent to cryopreserve MSC in the UC format for future extraction. Extraction of MSC from the UC often requires more-than-minimal manipulation and both the U.S. FDA and EMA stipulate the manufacturing of cell-based therapeutic products under cGMP conditions. To avoid disqualification of clinical use of MSC extracted under non-cGMP conditions, our laboratory stores MSC samples as minimally-manipulated sliced UC discs. Furthermore, it is anticipated that current bio-repositories of UC will benefit from future improvements in cell extraction and expansion.

The sub-amniotic membrane or UC lining (UCL), perivascular regions and Wharton's Jelly (WJ) of the UC are rich in MSC ${ }^{[5]}$. Unlike the WJ, UCL has been reported to also harbour epithelial stem cells. Our laboratory had performed the explant method to extract and expand MSC from cryopreserved WJ for cGMP development. However, tissue explantation is tedious and does not allow for concurrent sample processing. Unlike the UCL which has been reported to generate 20 million MSC at first passage ${ }^{[6]}$, we experienced inconsistent rates of MSC outgrowth from explanted WJ. To improve on the efficiency of MSC extraction from cryopreserved WJ, we surveyed literature for mechanical dissociation (grinding) and enzymatic dissociation (digestion). However, studies which use cryopreserved WJ as the starting material are scant and we note the one by Fong et al $2015^{[7]}$.
Consequently, we investigated if mechanical or enzymatic dissociation of MSC from cryopreserved WJ is superior over the explant method.

This study is outlined in Figure 1. UC were collected from both vaginal deliveries and Caesarean sections, with gestational ages ranging from $37-42$ weeks. Samples were transported dry in sterile polyethylene canisters, whereupon reaching the laboratory, were soaked with disinfectant solution (1\% antibiotic and anti-mycotic solution in DPBS) for 1 hour. Samples were sliced into $0.5 \mathrm{~cm}$ thick discs and rinsed repeatedly with the disinfectant solution. $2 \mathrm{~cm}$ worth of UC discs were transferred into each cryovial and soaked in cryoprotectant solution, consisting of DMEM: F12 supplemented with $10 \%$ DMSO, for 10 mins. All samples underwent controlled rate freezing between 24 to 48 hours from collection and were stored at $196^{\circ} \mathrm{C}$ for at least 7 days before thawing at $37^{\circ} \mathrm{C}$. Thawed UC discs were rinsed with DPBS and laid out on a petri dish. The WJ was carefully excised away from the UCL. $1 \mathrm{~cm}$ of UC produced $0.59 \pm$ $0.08 \mathrm{~g}$ of $\mathrm{WJ}$, which was minced into $2-5 \mathrm{~mm}$ pieces to maximise surface area, before treatment with the methods under investigation.

For the explant method, $40-60$ minced WJ pieces were spotted on a $10 \mathrm{~cm}$ culture dish and left to dry for 30 mins to promote adherence. $8 \mathrm{ml}$ of complete culture media, consisting of DMEM (low glucose formulation) supplemented with 10\% MSC growth-certified FBS, 1\% L-glutamine and $1 \%$ antibiotic and anti-mycotic solution, was then added to the dish. For the mechanical dissociation method, a disposable tissue grinder (VWR International) was used. Minced WJ was transferred to the grinder apparatus together with $2 \mathrm{ml}$ of complete culture media, followed by manipulation of the pestle for 5 mins at room temperature. The resultant tissue suspension was plated on a 10 $\mathrm{cm}$ dish with the addition of $8 \mathrm{ml}$ of complete culture media. For the enzyme dissociation method, minced WJ was incubated with a proprietary blend of enzymes for 2 hours at $37^{\circ} \mathrm{C}$, with shaking. The mixture was washed twice with complete culture media and seeded into a T25 flask with a culture volume of $5 \mathrm{ml}$. All of the cultures were kept at $37^{\circ} \mathrm{C}$ in a humidified $5 \% \mathrm{CO}_{2}$ incubator.

Author Names in full: Peng Yew Kenny Boey ${ }^{1 \#}$, Say Liang Daniel Lim ${ }^{1 \#}$, Kin Fai Tang ${ }^{1}$, Ming Ming Li ${ }^{1}$, Andrew Krishna Ekaputra ${ }^{1}$, Prosanto Kumar Chowdhury $^{1}$, Rajat Anand Gopal Mukherjee ${ }^{1}$, Jennifer Teo ${ }^{1}$, Arvin C. Faundo ${ }^{1}$ and Yoke Fong Chiew ${ }^{1}$

${ }^{1}$ Cordlife Group Limited

"These authors contributed equally to this work 


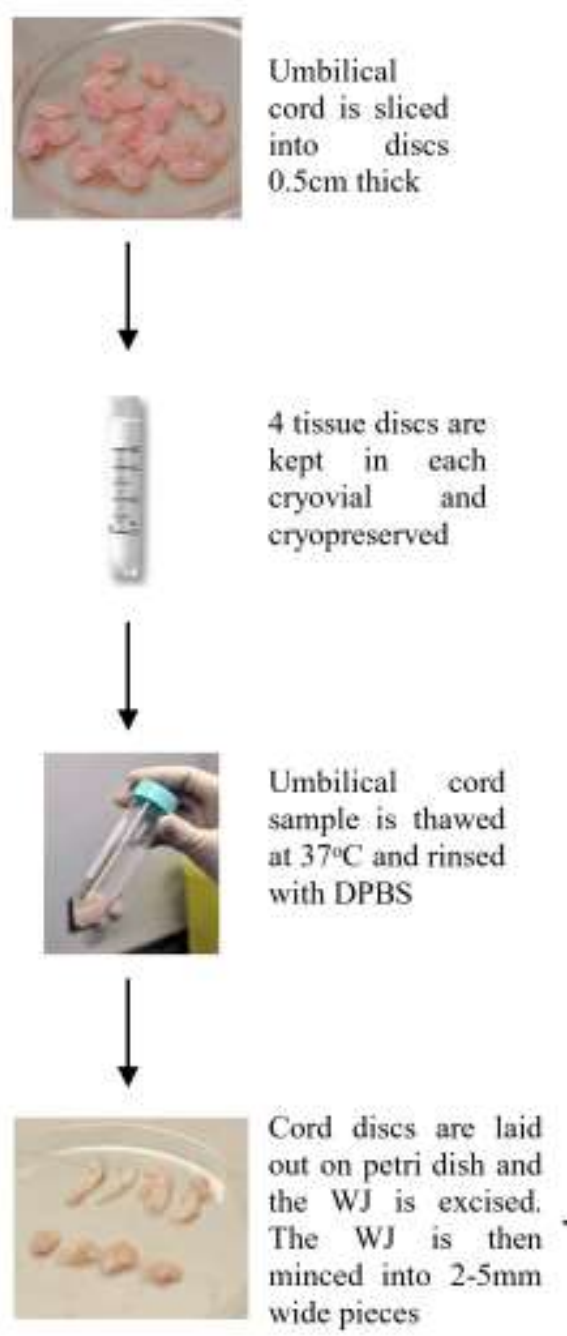

wide pieces
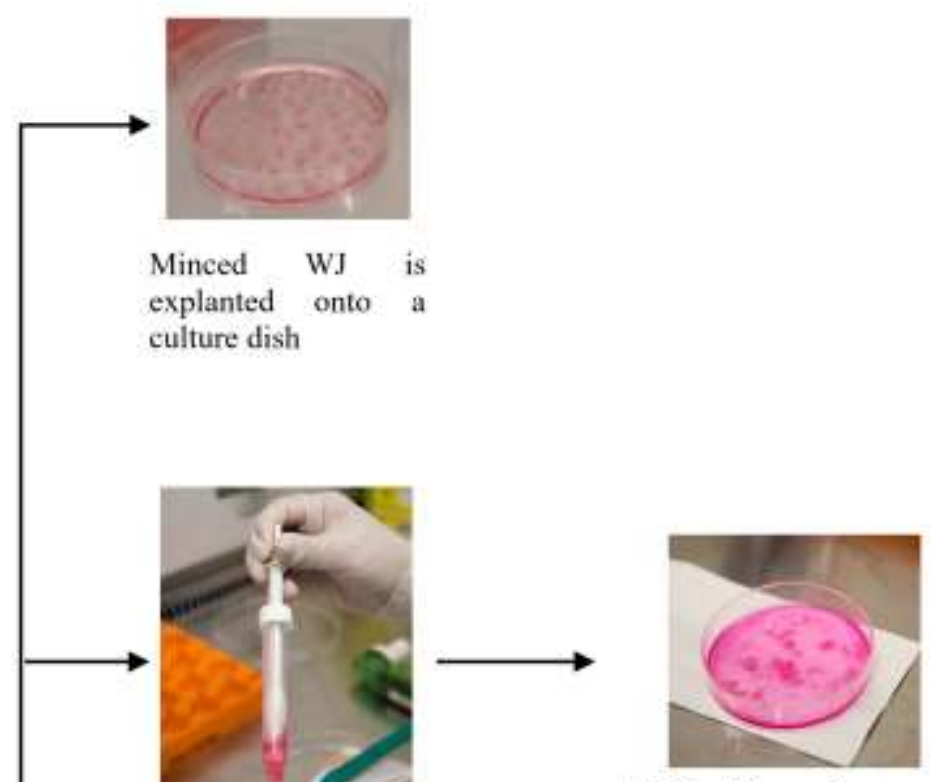

Grinded tissue fragments are seeded in a culture dish
Minced WJ is grinded in a sterile, single-use grinder apparatus

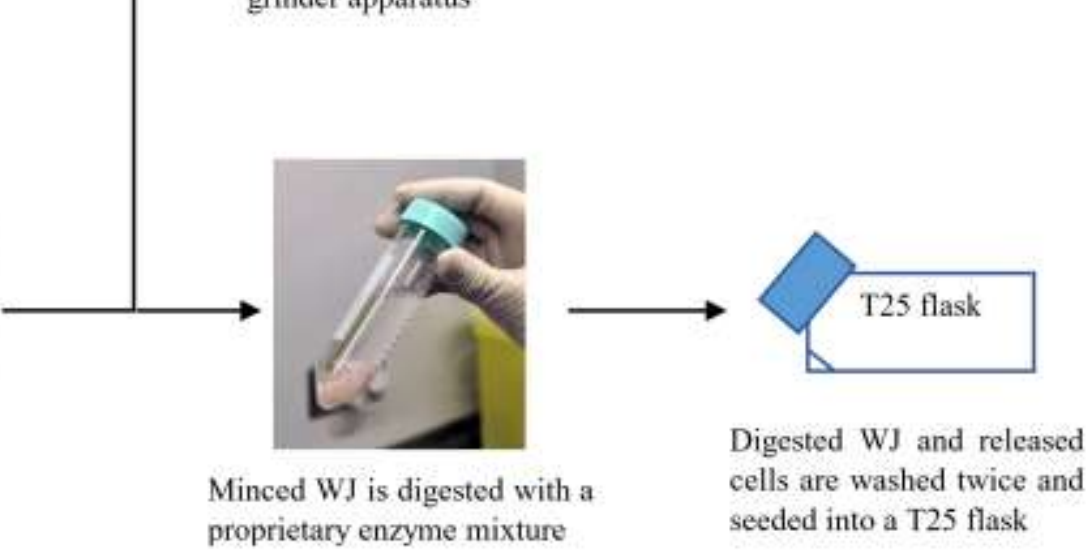

Figure 1. Methods investigated in this study for the extraction of MSC from cryopreserved WJ. Fresh UC was disinfected with antibiotic/anti-mycotic solution before cryopreservation. After thawing, the WJ was dissected from the UC. The dissected material was either explanted, grinded or treated with enzymes. Cells released from the former 2 methods were seeded in $10 \mathrm{~cm}$ culture dishes, while enzymatically-released cells were cultured in a T25 flask. At least 5 samples were processed for each method.

The yield of MSC was not immediately assessed post-grinding or digestion because the suspensions would include remnant cord blood cells. Instead, cells were cultured until sizable colonies were observed. Unlike Chatzistamatiou and colleagues ${ }^{[8]}$, we and Fong et $a l^{[7]}$ experienced success in extracting MSC from cryopreserved $\mathrm{WJ}$, and we speculate that this was due to the differences in cryopreservation protocol. Cultures from the enzyme digests reached confluency at 15.6 days (SD 3.9, $\mathrm{N}=13$ ), 3.1 times faster than the explant method, which required 48.7 days (SD 19.1, N =34). Notably, mechanical dissociation fared poorly as no adherent cells were observed $(\mathrm{N}=5)$. Cultures were rinsed with PBS to remove non-adherent cells, followed by incubation with $0.25 \%$ trypsin solution to detach the adherent MSC and enumerated by Trypan Blue exclusion assay. There was no significant difference between the average cell yields from cultures resultant from the digest method $\left(2.06 \times 10^{5}\right.$ cells $)$ and explant method $\left(2.38 \times 10^{5}\right.$ cells $), \mathrm{p}=0.69(\mathrm{t}-$ test). Because the digest method releases MSC in suspension, cells immediately gain access to nutrients and substrate to grow upon, resulting in improved yield over the explant method ${ }^{[9]}$. Enzymatic digestion, but not tissue explantation or grinding, presents efficiency and consistency as it allows simultaneous processing of multiple $\mathrm{WJ}$ samples to produce confluent cultures in a predictable timeframe. Tissue grinding either failed to extract sufficient MSC from cryopreserved $\mathrm{WJ}$ for initial cell seeding or was too injurious to the released cells. The extraction-dependant failure was corroborated by outgrowth of MSC from biologically-matched samples which were explanted instead. We had reduced the grinding duration and used non-cryopreserved UC to optimize the process but received limited success with the cell yield (data not shown).

The explant and digest methods both gave rise to adherent spindlelike cells, without any discernible differences in morphology (Figure 2A). To ascertain that the cells from the explant and enzyme digest methods were indeed MSC, they were cultured to sufficient numbers for flow cytometric analysis. All samples co-expressed CD73, CD90 and CD105, while remaining negative for the hematopoietic lineage cell markers: CD45, CD34, CD11b, CD19 and HLA-DR. MSC from explant and enzyme digests had comparable biomarker expression profiles (data not shown). Altogether, the results indicate that both cryopreservation and enzymatic digestion of WJ do not negatively affect the characteristics of the MSC within. 
A
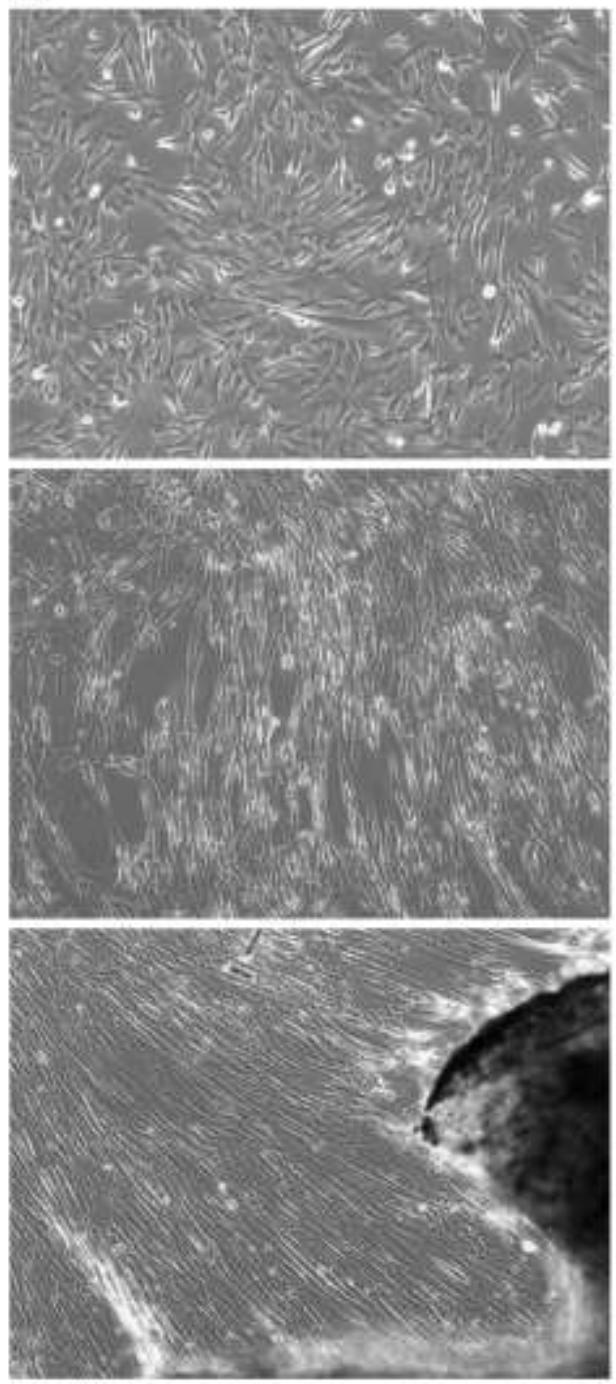

B
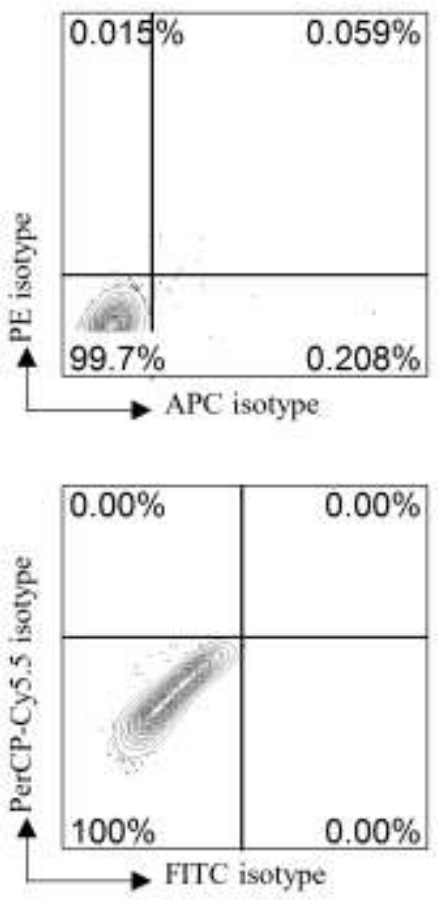
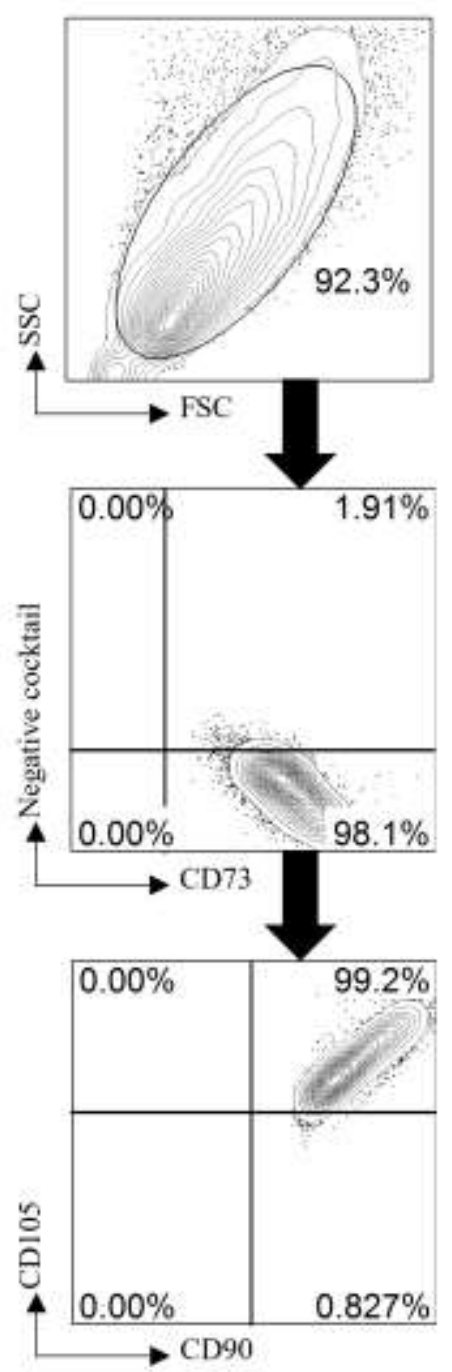

Figure 2. Characterization of MSC extracted from the WJ with the enzyme digestion method. (A) Morphology of MSC derived from a representative cryopreserved WJ sample under different conditions, at 100× total magnification. Top panel, 1-week culture of MSC extracted by enzymatic digestion. Middle panel, sub-culture of cells from the digest. These sub-cultured cells (passage 1) remained spindle-like in appearance and were used for immuno-fluorescence staining. Bottom panel, outgrowth of MSC from the same sample which was explanted for 29 days. (B) Flow cytometric analysis of MSC extracted by enzymatic digestion at passage 1. MSC were stained for CD73-APC, CD90-FITC and CD105-PerCPCy5.5, along with their relevant isotype controls, using the Becton Dickinson Stemflow hMSC analysis kit. They were also stained for markers associated with hematopoietic lineage cells, using a Negative Cocktail of PE-conjugated antibodies specific for CD45, CD34, CD11b, CD19 and HLA-DR. Cells were gated for by their forward and side scatter (FSC/SSC) profile to exclude debris and doublets. The gated cells were selected for their expression of CD73 and absence of hematopoietic lineage cell markers mentioned earlier. The majority of these sub-gated cells were found to co-express CD90 and CD105. The relevant isotype controls (isotype) are depicted on the left of each plot. The matched explanted sample was also analysed through flow cytometry and displayed an identical MSC biomarker expression profile (data not shown). Representative data from 1 WJ sample is shown.

\section{References}

1. Pittenger MF, Mackay AM, Beck SC, Jaiswal RK, Douglas R, Mosca JD, Moorman MA, Simonetti DW, Craig S, Marshak DR. Multilineage potential of adult human mesenchymal stem cells. Science 1999; 284(5411):143-7.

2. Nardi NB, da Silva Meirelles L. Mesenchymal stem cells: isolation, in vitro expansion and characterization. Stem cells: Springer; 2008. p. 249-82.

3. Dominici M, Le Blanc K, Mueller I, Slaper-Cortenbach I, Marini F, Krause D, Deans R, Keating A, Prockop D, Horwitz E. Minimal criteria for defining multipotent mesenchymal stromal cells. The International Society for Cellular Therapy position statement. Cytotherapy 2006;8(4):315-7.

4. Baksh D, Yao R, Tuan RS. Comparison of proliferative and multilineage differentiation potential of human mesenchymal stem cells derived from umbilical cord and bone marrow. Stem cells 2007;25(6):1384-92.

5. Nagamura-Inoue T, He H. Umbilical cord-derived mesenchymal stem cells: Their advantages and potential clinical utility. World J Stem Cells 2014;6(2):195-202.
6. Lim IJ, Phan TT. Epithelial and mesenchymal stem cells from the umbilical cord lining membrane. Cell Transplant 2014;23(45):497-503.

7. Fong CY, Subramanian A, Biswas A, Bongso A. Freezing of Fresh Wharton's Jelly From Human Umbilical Cords Yields High Post-Thaw Mesenchymal Stem Cell Numbers for CellBased Therapies. J Cell Biochem. 2016;117(4):815-27.

8. Chatzistamatiou TK, Papassavas AC, Michalopoulos E, Gamaloutsos C, Mallis P, Gontika I, Panagouli E, Koussoulakos SL, Stavropoulos-Giokas C. Optimizing isolation culture and freezing methods to preserve Wharton's jelly's mesenchymal stem cell (MSC) properties: an MSC banking protocol validation for the Hellenic Cord Blood Bank. Transfusion. 2014;54(12):3108-20.

9. Ding DC, Chang YH, Shyu WC, Lin SZ. Human umbilical cord mesenchymal stem cells: a new era for stem cell therapy. Cell Transplant. 2015;24(3):339-47. 
Chiew YF, et al. J Stem Cells Regen Med 2017; 13(1)

\author{
Abbreviations \\ cGMP: $\quad$ current Good Manufacturing Practice \\ FBS: $\quad$ Fetal Bovine Serum \\ FDA: $\quad$ Food and Drug Administration \\ DMSO: Dimethyl Sulfoxide \\ DPBS: Dulbecco's Phosphate Buffered Saline \\ DMEM:F12:Dulbecco's Modified Eagle's Medium: Nutrient Mixture F-12 \\ EMA: $\quad$ European Medicines Agency
}

\title{
Potential Conflicts of Interests
}

All the authors of this article are employees of Cordlife Group Limited, Singapore.

\section{Sponsors/grants}

This study was funded entirely by Cordlife Group Limited, a multi-product healthcare company based in Singapore.

\section{Corresponding Author}

Chiew Yoke Fong, Cordlife Group Limited, 1 Yishun Industrial Street 1, A’Posh Bizhub, \#06-01, Singapore 768160; E-mail: drchiew@cordlife.com 